\title{
Characterization of Yersinia enterocolitica and Yersinia pseudotuberculosis by Deoxyribonucleic Acid Hybridization and by Biochemical Reactions
}

DON J. BRENNER, ARNOLD G. STEIGERWALT, DEISE P. FALCÃO,' ROBERT E. WEAVER, AND G. RICHARD FANNING ${ }^{2}$

Center for Disease Control, Atlanta, Georgia 30333

\begin{abstract}
Strains of Yersinia enterocolitica and Yersinia pseudotuberculosis were characterized by deoxyribonucleic acid (DNA) hybridization, and the extent of DNA relatedness between the strains was assessed by hydroxyapatite chromatography. DNAs from 24 strains of $Y$. pseudotuberculosis were highly related, but $Y$.pseudotuberculosis was only 40 to $60 \%$ related to $Y$. enterocolitica. $Y$. enterocolitica strains formed three DNA relatedness groups, and the data strongly suggest a fourth relatedness group. Each relatedness group can be defined biochemically. One DNA relatedness group corresponds to typical $Y$. enterocolitica; the second is rhamnose positive; the third is rhamnose positive, melibiose positive, $\alpha$-methyl glucoside positive, and raffinose positive. The suggested fourth hybridization group is sucrose negative. Although all of these DNA relatedness groups should remain in the genus Yersinia, only the first group is $Y$. enterocolitica. All of the yersiniae tested are distantly, but significantly, related to other members of Enterobacteriaceae. Yersiniae were more related to members of Enterobacteriaceae than to Pasteurella multocida.
\end{abstract}

The former Pasteurella species $P$. pestis and $P$. pseudotuberculosis have been placed in the new genus Yersinia within the family Enterobacteriaceae (9). A third species, Yersinia enterocolitica, has also been placed in this genus $(9,13,25)$. Y. enterocolitica was first described in the United States in 1939 (30) and then in Europe some 10 years later (16). The organisms were human pathogens described as resembling Yersinia pseudotuberculosis or aberrant forms of $Y$. pseudotuberculosis (16, 30). A fourth species, Yersinia philomiragia, was proposed in 1969 (18), but it has not been generally accepted.

In the $1950 \mathrm{~s}$ and $1960 \mathrm{~s}, Y$. enterocolitica was isolated from various animal species, including the chinchilla, cat, dog, galago, deer, cow, beaver, monkey, Canada goose, camel, racoon, robin, oyster, pig, and hare, and also from water $(20,24,34,37,39)$. Until 1966 there were only 23 isolates of $Y$. enterocolitica from humans; by 1974 this figure had soared to more than 4,000 (34). Most of the isolates were from Europe; the first culturally confirmed case in the United States in 21 years was reported in 1970 (32). These organisms have been implicated in acute

\footnotetext{
${ }^{1}$ Present address: Faculdade de Farmácia e Odontologia de Araraquara, Rua Expedicionários do Brasil, 1621-Calxa Postal, 331, Araraquara 14.800.S.P. Brazil.

${ }^{2}$ Present address: Walter Reed Army Institute of Research, Washington, D.C. 20012.
}

gastroenteritis, acute terminal ileitis, mesenteric lymphadenitis, septicemia, conjunctivitis, meningitis, abscesses, polyarthritis, and Crohn's disease $(1,15,17,23,27,32,34,36,39)$.

$Y$. pseudotuberculosis strains are biochemically homogeneous $(24,37,38)$, whereas the biochemical reactions given by strains of $Y$. enterocolitica are quite variable $(2,10,19,26$, 31, 33-36; G. Wauters, Ph.D. thesis, Catholic Univ. of Louvain, Vander, Louvain, Belgium, 1970). Y. enterocolitica strains separate into at least two groups when tested by numerical taxonomy (33). The species has been divided into four or five bio-groups on the basis of reactions for indole, xylose, esculin, and salicin (19, 26; G. Wauters, Ph.D. thesis). Several authors $(2,19,25,33-35)$ have discussed the possibility that strains of $Y$. enterocolitica should be split into two or more species. Among the atypical strains are rhamnose-positive organisms $(2,10$, $19,33,36)$ and sucrose-negative organisms (10, $19,26,35,36$ ).

The International Committee on Systematic Bacteriology Subcommittee on the Taxonomy of Pasteurella, Yersinia, and Franciscella has recognized the need for genetic studies (25). Thus far, the guanine plus cytosine $(\mathrm{G}+\mathrm{C})$ contents of yersiniae have been determined (35), a partial genetic map of $Y$. pseudotuberculosis has been established by interrupted mating experiments (22), and preliminary deoxyribonu- 
cleic acid (DNA) hybridization experiments have been carried out by using single strains of $Y$. pseudotuberculosis, Y. pestis, Y. phlomiragia, and pasteurellae (28).

The purpose of this study was to characterize $Y$. enterocolitica and $Y$. pseudotuberculosis both biochemically and by DNA hybridization.

DNA relatedness and the phenotypic basis of DNA relatedness are determined. The data are discussed with regard to biogrouping, speciation, and diagnostic identification of yersiniae.

\section{MATERIALS AND METHODS}

Organisms, media, and biochemical characterization. The bacteria used in this study are listed in Table 1. The organisms were maintained on either brain heart infusion agar or blood agar base. Biochemical tests were carried out as described by Edwards and Ewing (11). Deoxyribonuclease was assayed as described by Ewing and Davis (12). All tests were carried out at $35 \mathrm{C}$ except that for gelatin, which was incubated at 22 or $35 \mathrm{C}$ and then refrigerated to assay for liquefaction, and that for motility, which was carried out at 22 as well as 35 C. Except where contraindicated (11), tests were held and read for 7 days.

Bacteria were cultivated on brain heart infusion broth, and a modified $\mathrm{C}$ medium was used in the preparation of DNA labeled with ${ }^{32} \mathrm{PO}_{4}$ (5). The preparation of both labeled and unlabeled DNA has been described (5).

DNA reassociation. The conditions used for DNA reassociation, the subsequent separation of singleand double-stranded DNA on hydroxyapatite (HA), and thermal elution chromatography on HA have been described previously (6-8). DNA from reference organisms was labeled with ${ }^{32} \mathrm{PO}_{4}$. The labeled DNA at a concentration of $0.1 \mu \mathrm{g} / \mathrm{ml}$ (specific activity of between $1 \times 10^{4}$ counts/min per $\mu \mathrm{g}$ and $1.2 \times$ $10^{5}$ counts/min per $\mu \mathrm{g}$ ) was added to $150 \mu \mathrm{g}$ of unlabeled DNA per $\mathrm{ml}$ from the homologous strain and other strains of interest. The 1,500 -fold excess of unlabeled DNA insured maximum opportunity for the labeled DNA to reassociate with unlabeled DNA and little opportunity for complementary strands of labeled DNA to reassociate. The mixture of labeled and unlabeled DNA in $0.28 \mathrm{M}$ phosphate buffer (PB; equimolar mixture of $\mathrm{Na}_{2} \mathrm{HPO}_{4}$ and $\mathrm{NaH}_{2} \mathrm{PO}_{4}$ ) was heat denatured and incubated at the desired temperature for $16 \mathrm{~h}$ (approximately $100 \mathrm{C}_{0} \mathrm{t}$ 's; reference 8). Self-reaction of labeled DNA was controlled by including a reaction containing only labeled DNA. After incubation, the mixture was diluted to $0.14 \mathrm{M} \mathrm{PB}$ and passed through HA equilibrated with $0.14 \mathrm{M} \mathrm{PB}+0.4 \%$ sodium dodecyl sulfate. Reassociated (double-stranded, hybridized) DNA was adsorbed to HA and single-stranded DNA was eluted. The double-stranded DNA was eluted by either increasing the PB concentration to $0.4 \mathrm{M} \mathrm{PB}$ or by increasing the temperature in 5-degree increments. In the latter case, bound DNA became denatured and eluted from HA as the temperature exceeded the $T_{m(\rho)}$ (that temperature at which $50 \%$ of a given double-stranded DNA was eluted from HA).

The amount of relatedness between organisms was assessed by normalizing the amount of DNA bound to HA in heterologous reactions to that bound to HA in the homologous control reaction. Relatedness was expressed in percent and is conveniently referred to as the relative binding ratio (RBR). Percent divergence (D) of the related sequences can be calculated by comparing the thermal stability of the heterologous reactions to that of the homologous reaction. Approximately 1\% unpaired bases was assumed to result in a 1-degree decrease in thermal stability (21). Reactions were carried out both at $60 \mathrm{C}$, where optimum reassociation occurs, and at $75 \mathrm{C}$, where only closely related sequences will reassociate. In these studies the average homologous DNA reassociation was between 75 and $90 \%$, and the binding of a control containing only labeled DNA was 1 to $3 \%$. This control value was subtracted from reactions exhibiting less than $10 \%$ reassociation. Closely or highly related strains were usually taken as those that yield an RBR of $70 \%$ or greater. Characteristic of closely related strains is the fact that the majority of DNA sequences that form duplexes at $60 \mathrm{C}$ are stable at $75 \mathrm{C}$.

\section{RESULTS}

Strains of $Y$. enterocolitica and $Y$. pseudotuberculosis obtained from diverse geographical areas and from human, animal, and water sources were employed to obtain a representative sample (Table 1). Strains that were atypical biochemically were included in an attempt to define the taxonomic boundaries of these species. Approximately 65 biochemical tests were used to characterize most of the strains studied (Tables 2 and 3). Y. enterocolitica strains gave both positive and negative reactions in 31 tests (Table 2), whereas Y. pseudotuberculosis strains gave both positive and negative reactions in only 10 tests (Table 3 ).

DNA hybridization reactions were carried out to group these yersiniae genotypically, to determine genetic relatedness between these yersiniae and other members of Enterobacteriaceae, and to correlate phenotypic heterogeneity with genetic divergence. Table 4 shows the data obtained by using labeled DNA from $Y$. enterocolitica 501-70. In $60 \mathrm{C}$ reactions, 13 strains of $Y$. enterocolitica had RBR values of 85 to $100 \%$ to strain $501-70$. The percent $\mathrm{D}$ of the related sequences was 0 to 2.4. Further evidence of the close relatedness of these strains are the RBR values of 82 to $100 \%$ obtained in reactions carried out at the more restrictive $75 \mathrm{C}$ criterion. A single strain of $Y$. pestis gave an RBR of $43 \%$ to strain 501-70. In this instance the related sequences showed a percent $\mathrm{D}$ of 15.2 , and the RBR dropped to $11 \%$ at $75 \mathrm{C}$. Four strains of Red Mouth bacterium, a fish pathogen (29), were $30 \%$ related to $Y$. enterocolitica 
TABLE 1. Bacterial strains tested

\begin{tabular}{|c|c|c|}
\hline Strain & Origin & Received from \\
\hline Arizona hinshawii & & $\mathrm{CDC}^{a}$ \\
\hline Citrobacter freundii $80-58$ & & CDC \\
\hline Edwardsiella tarda $483-59$ & & CDC \\
\hline E. tarda $3979-60$ & & $\mathrm{CDC}$ \\
\hline E. tarda $4830-60$ & & CDC \\
\hline Enterobacter aerogenes $1627-66$ & & $\mathrm{CDC}$ \\
\hline Enterobacter agglomerans $1599-71$ & & $\mathrm{CDC}$ \\
\hline E. agglomerans 14589 & & $\mathrm{ATCC}^{b}$ \\
\hline Enterobacter cloacae 121-65 & & $\mathrm{CDC}$ \\
\hline E. cloacae $256-4$ & & V. Brenner \\
\hline E. cloacae $1347-71$ & & CDC \\
\hline Enterobacter hafniae $932-68$ & & CDC \\
\hline Erwinia amylovora EA 178 & & $\mathrm{ICPB}^{c}$ \\
\hline Escherichia coli $\mathrm{K}-12$ & & U. of Washington \\
\hline Klebsiella pneumoniae 2 & & CDC \\
\hline Pasteurella multocida 6530 & & ATCC \\
\hline P. multocida 6535 & & ATCC \\
\hline P. multocida 6587 & & ATCC \\
\hline P. multocida 7707 & & ATCC \\
\hline P. multocida 7773 & & ATCC \\
\hline P. multocida 7960 & & ATCC \\
\hline P. multocida 7987 & & ATCC \\
\hline P. multocida 8369 & & ATCC \\
\hline P. multocida 9556 & & ATCC \\
\hline P. multocida 9658 & & ATCC \\
\hline P. multocida 10544 & & ATCC \\
\hline P. multocida 12947 & & ATCC \\
\hline P. multocida 12948 & & ATCC \\
\hline P. multocida 15742 & & ATCC \\
\hline P. multocida 15743 & & ATCC \\
\hline Pectobacterium carotovorum 495 & & ATCC \\
\hline Proteus mirabilis 1 & & S. Falkow \\
\hline Proteus morganii & & S. Falkow \\
\hline Proteus vulgaris PR 1 & & CDC \\
\hline Red Mouth 2396-61 & & $\mathrm{CDC}$ \\
\hline Red Mouth 2514-63 & & $\mathrm{CDC}$ \\
\hline Red Mouth 4535-69 & & CDC \\
\hline Red Mouth 5972-61 & & CDC \\
\hline Salmonella typhimurium LT2 & & T. Theodore \\
\hline Serratia marcescens SM6 red & & WRAIR $^{d}$ \\
\hline Shigella flexneri 24570 & & S. Falkow \\
\hline Shigella sonnei virulent & & WRAIR \\
\hline Yersinia enterocolitica 17 & & B. Chester \\
\hline Y. enterocolitica 39 & Chinchilla, Holland & L. LeMinor \\
\hline$Y$. enterocolitica 48 & & B. Chester \\
\hline$Y$. enterocolitica 56 & & B. Chester \\
\hline Y. enterocolitica 57 & & B. Chester \\
\hline$Y$. enterocolitica 64 & Chinchilla, Switzerland & L. LeMinor \\
\hline Y. enterocolitica 75 & Chinchilla, Switzerland & L. LeMinor \\
\hline Y. enterocolitica 96 & Chinchilla, Denmark & L. LeMinor \\
\hline Y. enterocolitica 97 & Chinchilla, Denmark & L. LeMinor \\
\hline Y. enterocolitica 102 & Human, Denmark & L. LeMinor \\
\hline Y. enterocolitica 103 & Sheep, Faroe Island & L. LeMinor \\
\hline Y. enterocolitica 105 & Human, Denmark & L. LeMinor \\
\hline Y. enterocolitica 106 & Guinea pig, Denmark & L. LeMinor \\
\hline Y. enterocolitica 107 & Human, United States & L. LeMinor \\
\hline Y. enterocolitica 123 & Cow, France & L. LeMinor \\
\hline Y. enterocolitica 124 & Horse, France & L. LeMinor \\
\hline Y. enterocolitica 135 & Chinchilla, Holland & L. LeMinor \\
\hline Y. enterocolitica 160 & Human, Switzerland & L. LeMinor \\
\hline Y. enterocolitica 161 & Human, United States & L. LeMinor \\
\hline$Y$. enterocolitica 175 & Human, Belgium & L. LeMinor \\
\hline Y. enterocolitica 178 & Hare, England & L. LeMinor \\
\hline Y. enterocolitica $201-75$ & Human, United States & CDC \\
\hline Y. enterocolitica 211 & Human, Belgium & L. LeMinor \\
\hline Y. enterocolitica 373 & Human, Finland & L. LeMinor \\
\hline Y. enterocolitica 480 & Human, Belgium & L. LeMinor \\
\hline Y. enterocolitica 490 & Hare, France & L. LeMinor \\
\hline
\end{tabular}


TABLE 1-Continued

\begin{tabular}{|c|c|c|}
\hline Strain & Origin & Received from \\
\hline Y. enterocolitica 495-70 & Human, United States & $\mathrm{CDC}$ \\
\hline$Y$. enterocolitica $496-70$ & Monkey, United States & $\mathrm{CDC}$ \\
\hline Y. enterocolitica 497-70 & Monkey, United States & $\mathrm{CDC}$ \\
\hline Y. enterocolitica $498-70$ & Human, United States & CDC \\
\hline Y. enterocolitica $499-70$ & Human, United States & CDC \\
\hline Y. enterocolitica 500 & Hare, France & L. LeMinor \\
\hline Y. enterocolitica $500-70$ & Human, United States & $\mathrm{CDC}$ \\
\hline Y. enterocolitica $501-70$ & Monkey, United States & CDC \\
\hline Y. enterocolitica $502-70$ & Human, Belgium & $\mathrm{CDC}$ \\
\hline Y. enterocolitica $503-70$ & Human, United States & CDC \\
\hline Y. enterocolitica 504-70 & Human, Sweden & CDC \\
\hline Y. enterocolitica $505-70$ & Human, Sweden & CDC \\
\hline Y. enterocolitica 506-70 & Human, Sweden & CDC \\
\hline$Y$. enterocolitica 551 & Human, Belgium & L. LeMinor \\
\hline$Y$. enterocolitica 614 & Hare, Holland & L. LeMinor \\
\hline$Y$. enterocolitica 842 & Human, United States & L. LeMinor \\
\hline$Y$. enterocolitica 845 & Human, United States & L. LeMinor \\
\hline$Y$. enterocolitica 846 & Human, United States & L. LeMinor \\
\hline Y. enterocolitica 867 & Human, Belgium & L. LeMinor \\
\hline$Y$. enterocolitica 955 & Water, Norway & L. LeMinor \\
\hline$Y$. enterocolitica 1110 & Human, United States & L. LeMinor \\
\hline Y. enterocolitica 1367 & Human, Czechoslovakia & L. LeMinor \\
\hline$Y$. enterocolitica 1474 & Water, Norway & L. LeMinor \\
\hline Y. enterocolitica 1475 & Water, Norway & L. LeMinor \\
\hline$Y$. enterocolitica 1477 & Water, Norway & L. LeMinor \\
\hline Y. enterocolitica C 1889 & Water, United States & $\mathrm{CDC}$ \\
\hline Y. enterocolitica D 2705 & Human, United States & CDC \\
\hline Y. enterocolitica C 3651 & Human, United States & CDC \\
\hline$Y$. enterocolitica C 3741 & Human, United States & $\mathrm{CDC}$ \\
\hline Y. enterocolitica C 5188 & Human, United States & CDC \\
\hline Y. enterocolitica C 5427 & Human, United States & CDC \\
\hline Yersinia pestis & & L. Corwin \\
\hline Yersinia pseudotuberculosis P7 & Hare, Sweden & E. Thal \\
\hline Y. pseudotuberculosis P25 & Hare, Sweden & E. Thal \\
\hline Y. pseudotuberculosis P27 & Hare, Sweden & E. Thal \\
\hline Y. pseudotuberculosis P32 & Hare, Sweden & E. Thal \\
\hline Y. pseudotuberculosis P43 & Mink, Sweden & E. Thal \\
\hline Y. pseudotuberculosis P48 & Hare, Sweden & E. Thal \\
\hline Y. pseudotuberculosis P52 & Mink, Sweden & E. Thal \\
\hline Y. pseudotuberculosis P65 & Mink, Germany & E. Thal \\
\hline Y. pseudotuberculosis P68 & Mink, Germany & E. Thal \\
\hline Y. pseudotuberculosis $\mathrm{P} 105$ & Mink, Denmark & E. Thal \\
\hline Y. pseudotuberculosis P198 & Hare, Sweden & E. Thal \\
\hline Y. pseudotuberculosis P262 & Hare, Sweden & E. Thal \\
\hline Y. pseudotuberculosis P279 & Hare, Sweden & E. Thal \\
\hline Y. pseudotuberculosis P281 & Swallow, Sweden & E. Thal \\
\hline Y. pseudotuberculosis P293 & Human, Germany & E. Thal \\
\hline Y. pseudotuberculosis P308 & Human, Germany & E. Thal \\
\hline Y. pseudotuberculosis P320 & Human, Germany & E. Thal \\
\hline Y. pseudotuberculosis P324 & Human, Germany & E. Thal \\
\hline Y. pseudotuberculosis P385 & Hare, France & E. Thal \\
\hline Y. pseudotuberculosis P407 & Hare, France & E. Thal \\
\hline Y. pseudotuberculosis P417 & Guinea pig, England & E. Thal \\
\hline Y. pseudotuberculosis P418 & Guinea pig, England & E. Thal \\
\hline Y. pseudotuberculosis P463 & Guinea, pig, Japan & E. Thal \\
\hline Y. pseudotuberculosis P907 & Guinea pig, England & ATCC \\
\hline Y. pseudotuberculosis $\mathrm{P} 913$ & Guinea pig, England & ATCC \\
\hline Y. pseudotuberculosis 4284 & Guinea pig, England & ATCC \\
\hline Y. pseudotuberculosis 23207 & Guinea pig, Germany & ATCC \\
\hline
\end{tabular}

${ }^{a}$ CDC, Center for Disease Control.

${ }^{b}$ ATCC, American Type Culture Collection.

c ICPB, International Collection of Phytopathogenic Bacteria.

${ }^{d}$ WRAIR, Walter Reed Army Institute of Research.

with $15 \% \mathrm{D}$. RBR values of $Y$. enterocolitica 501-70 and all other members of Enterobacteriaceae tested were 10 to $25 \%$. With tests at $75 \mathrm{C}$, these RBR values, as expected, decreased to $5 \%$ or less in $75 \mathrm{C}$ reactions. Relatedness between most members of Enterobacteriaceae and Y. enterocolitica was 15 to $20 \%$. This is very similar to the "core" level of relatedness seen 


\begin{tabular}{|c|c|c|c|c|c|c|c|c|c|c|c|c|c|c|c|c|c|c|c|c|c|c|c|c|c|c|c|}
\hline โ9ז & + & + & + & + & & + & & + & & & & & & & + & & & & & & & +1 & & & & & \\
\hline$\nabla Z I$ & + & + & + & + & 1 & + & + & + & 1 & 1 & + & + & 1 & 1 & + & + & + & 1 & + & 1 & + & + & 1 & + & 1 & + & 1 \\
\hline$\varepsilon z I$ & + & + & + & + & 1 & + & + & + & 1 & 1 & + & 1 & T & t & + & + & + & 1 & + & 1 & + & + & 1 & + & 1 & + & 7 \\
\hline $90 \mathrm{I}$ & + & + & + & + & 1 & + & + & + & + & 1 & + & 1 & + & 1 & + & 1 & + & 1 & + & 1 & + & + & + & + & + & + & 1 \\
\hline zoI & + & + & + & + & 1 & + & + & + & 1 & 1 & + & 1 & 1 & 1 & + & + & + & 1 & + & 1 & + & + & + & + & 1 & + & 1 \\
\hline $0 L-867$ & + & + & 1 & + & 1 & + & + & + & & 1 & + & & & + & + & + & + & 1 & + & & + & & & & & 1 & 1 \\
\hline LOI & + & + & + & 1 & 1 & + & + & + & 1 & 1 & + & 1 & 1 & + & + & + & + & 1 & + & 1 & + & + & 1 & + & 1 & 1 & 1 \\
\hline$\angle 6$ & + & + & + & 1 & 1 & + & + & + & I & 1 & + & 1 & 1 & 1 & + & + & + & 1 & + & 1 & + & + & + & + & + & + & 1 \\
\hline $0 L-90 \mathrm{~S}$ & + & + & 1 & 1 & 1 & + & 1 & + & & 1 & + & & & + & + & & & 1 & & & + & & & & & 1 & \\
\hline $0 L-\varepsilon 09$ & + & + & 1 & + & 1 & + & + & + & & 1 & + & & & 1 & + & + & + & 1 & + & & + & & & & & । & 1 \\
\hline $0 L-66 t$ & + & + & 1 & + & 1 & + & + & + & & 1 & + & & & 1 & + & 1 & + & 1 & + & & + & & & & & 1 & 1 \\
\hline $0 L-\angle 6 \mathrm{t}$ & + & + & 1 & 1 & 1 & + & + & + & & 1 & + & & & 1 & + & + & + & 1 & + & & + & & & & & 1 & 1 \\
\hline $0 L-967$ & + & + & 1 & 1 & I & + & + & + & & 1 & + & & & 1 & + & 1 & + & 1 & + & & + & & & & & 1 & 1 \\
\hline$\varepsilon \angle \varepsilon$ & 1 & + & 1 & 1 & 1 & + & + & + & 1 & 1 & + & 1 & I & 1 & + & 1 & + & 1 & + & 1 & + & + & + & +1 & 1 & + & 1 \\
\hline IIZ & 1 & + & + & 1 & 1 & + & + & + & 1 & 1 & + & 1 & I & 1 & + & + & + & 1 & + & 1 & + & + & + & + & 1 & + & 1 \\
\hline $09 \mathrm{t}$ & 1 & + & + & + & I & + & + & + & 1 & 1 & + & 1 & 1 & 1 & + & + & + & 1 & + & 1 & + & 1 & + & + & 1 & 1 & 1 \\
\hline 96 & 1 & + & + & + & 1 & + & 1 & + & 1 & 1 & + & 1 & 1 & 1 & + & 1 & + & 1 & + & 1 & + & + & 1 & + & 1 & 1 & 1 \\
\hline 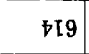 & 1 & 1 & + & + & 1 & + & + & + & 1 & I & + & 1 & I & 1 & + & 1 & + & 1 & + & 1 & 1 & + & + & + & 1 & + & 1 \\
\hline $0 L-70 \mathrm{~g}$ & 1 & 1 & 1 & + & 1 & + & + & + & & 1 & + & & & 1 & + & 1 & + & 1 & + & & + & & & & & 1 & 1 \\
\hline $0 L$ - I0S & 1 & + & 1 & + & 1 & + & + & + & & 1 & + & & & 1 & + & + & + & 1 & + & & + & & & & & 1 & 1 \\
\hline $0 L-00 \mathrm{~S}$ & 1 & + & 1 & + & 1 & + & + & + & & 1 & + & & & 1 & + & + & + & 1 & + & & + & & & & & 1 & 1 \\
\hline $0 L-96 \downarrow$ & 1 & + & 1 & + & 1 & + & + & + & & I & + & & & 1 & + & + & + & 1 & + & & + & & & & & $!$ & I \\
\hline $\mathrm{sL}_{\mathrm{T}} \mathrm{T} 0 \mathrm{Z}$ & 1 & + & 1 & 1 & 1 & + & + & + & + & 1 & + & 1 & 1 & 1 & + & + & + & 1 & + & & + & + & 1 & + & 1 & & \\
\hline $8 L \mathrm{I}$ & 1 & 1 & + & + & 1 & 1 & + & + & I & 1 & 1 & 1 & 1 & 1 & + & 1 & 1 & 1 & 1 & 1 & + & 1 & + & + & 1 & 1 & 1 \\
\hline SLI & $i$ & 1 & + & + & 1 & + & + & + & 1 & 1 & + & + & 1 & 1 & + & + & + & 1 & + & 1 & + & + & + & + & 1 & + & 1 \\
\hline$s \varepsilon \mathrm{L}$ & 1 & + & 1 & + & 1 & + & + & + & । & I & I & 1 & 1 & 1 & + & + & + & 1 & + & 1 & + & + & + & + & + & 1 & 1 \\
\hline$q_{L}$ & 1 & 1 & + & + & 1 & + & + & + & 1 & 1 & + & 1 & 1 & 1 & + & + & + & 1 & + & 1 & + & + & + & + & 1 & + & 1 \\
\hline 79 & i & + & + & 1 & 1 & + & + & + & 1 & I & + & 1 & 1 & 1 & + & 1 & + & 1 & + & 1 & + & + & + & + & 1 & + & 1 \\
\hline $6 \varepsilon$ & 1 & + & 1 & 1 & 1 & + & + & + & I & 1 & + & 1 & 1 & 1 & + & 1 & + & 1 & + & 1 & + & 1 & + & + & 1 & + & \\
\hline
\end{tabular}

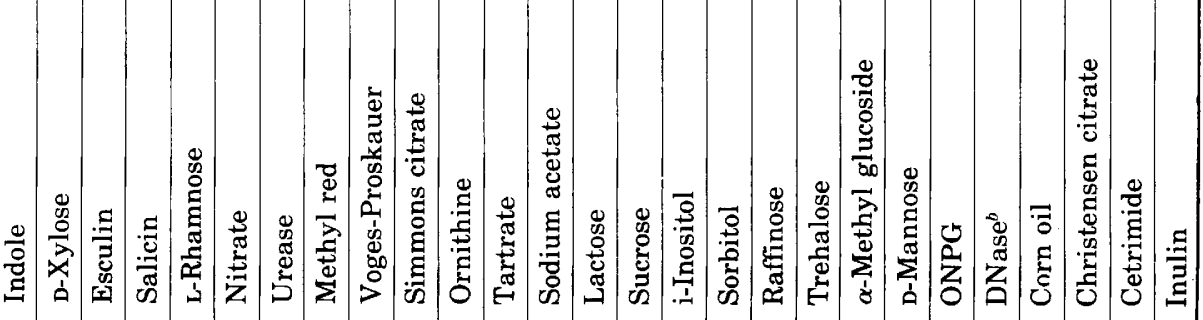




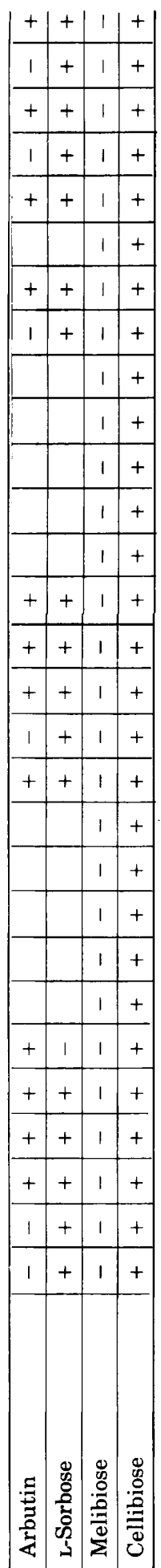

\begin{tabular}{|c|c|c|c|c|c|c|c|c|c|c|c|c|c|c|c|c|c|c|c|c|}
\hline LЪד9D & + & + & 1 & + & 1 & + & + & & & 1 & + & + & & 1 & + & I & + & 1 & + & \\
\hline 688 โก & + & + & 1 & + & I & + & + & & & 1 & + & + & I & + & + & 1 & + & 1 & + & \\
\hline GoLZa & + & + & + & + & + & + & + & + & 1 & + & + & + & 1 & + & + & 1 & + & 1 & + & 1 \\
\hline 88IGD & + & + & + & + & + & + & + & + & & 1 & + & & & + & + & + & + & 1 & + & \\
\hline โฤL\&อ & + & + & + & + & + & + & + & + & & 1 & + & + & 1 & 1 & + & + & + & + & + & + \\
\hline โ9980 & + & + & + & + & + & + & + & + & & I & + & + & 1 & + & + & & + & + & + & + \\
\hline$\angle G$ & + & + & + & + & + & + & + & 1 & & 1 & + & + & 1 & + & + & 1 & + & + & + & + \\
\hline 99 & + & + & + & + & + & + & + & 1 & & 1 & + & + & 1 & + & + & + & + & + & + & + \\
\hline 87 & + & + & + & + & + & + & + & 1 & & + & + & + & 1 & 1 & + & 1 & + & 1 & + & + \\
\hline$L I$ & + & + & + & + & + & + & + & 1 & & 1 & + & + & 1 & + & + & I & + & + & + & + \\
\hline 996 & + & + & + & + & + & + & + & + & 1 & + & + & + & 1 & + & + & + & + & + & + & + \\
\hline$\angle 98$ & + & + & + & + & + & + & + & + & 1 & 1 & + & + & 1 & I & + & + & + & 1 & + & I \\
\hline$G \angle D I$ & 1 & + & + & + & 1 & + & + & + & 1 & 1 & + & 1 & I & + & I & 1 & + & 1 & + & I \\
\hline$\nabla L D I$ & + & + & + & + & 1 & + & + & + & 1 & 1 & + & 1 & 1 & 1 & 1 & I & + & I & + & 1 \\
\hline $06 t$ & + & + & + & + & 1 & + & + & + & 1 & 1 & + & + & 1 & 1 & 1 & 1 & + & 1 & + & 1 \\
\hline 90I & + & + & + & + & 1 & + & + & + & 1 & 1 & + & I & 1 & + & 1 & 1 & + & 1 & + & 1 \\
\hline$\varepsilon 0 I$ & 1 & + & + & + & 1 & + & + & + & 1 & 1 & + & I & 1 & 1 & 1 & 1 & + & 1 & + & 1 \\
\hline$\angle L B I$ & + & + & + & + & 1 & + & + & + & 1 & 1 & + & I & 1 & 1 & + & + & + & 1 & + & 1 \\
\hline 087 & + & + & + & + & 1 & + & + & + & 1 & I & + & + & 1 & 1 & + & 1 & + & 1 & + & 1 \\
\hline$\angle 9 \& \mathrm{I}$ & + & + & + & + & 1 & + & + & + & I & I & + & + & + & + & + & + & + & 1 & + & 1 \\
\hline OIII & + & + & + & + & 1 & + & + & + & 1 & 1 & + & 1 & 1 & + & + & + & + & 1 & + & I \\
\hline 978 & + & + & + & + & 1 & + & + & + & 1 & 1 & + & 1 & I & + & + & + & + & 1 & + & I \\
\hline $\mathbf{9 \nsubseteq 8}$ & + & + & + & + & 1 & + & + & + & I & I & + & 1 & 1 & I & + & 1 & + & 1 & + & 1 \\
\hline Z¥8 & + & + & + & + & 1 & I & + & + & 1 & 1 & + & 1 & 1 & + & + & + & + & + & + & 1 \\
\hline ISG & + & + & + & + & 1 & + & + & 1 & 1 & 1 & + & 1 & 1 & + & + & + & + & 1 & + & 1 \\
\hline $0 L-G 0 S$ & + & + & + & + & 1 & + & + & + & & I & + & & & 1 & + & & + & 1 & + & \\
\hline $0 L-\mp 0 \mathrm{~g}$ & + & + & + & + & 1 & + & + & + & & 1 & + & & & 1 & + & & + & 1 & + & \\
\hline 009 & + & + & + & + & 1 & + & + & + & 1 & 1 & + & + & 1 & 1 & + & + & + & 1 & + & \\
\hline
\end{tabular}




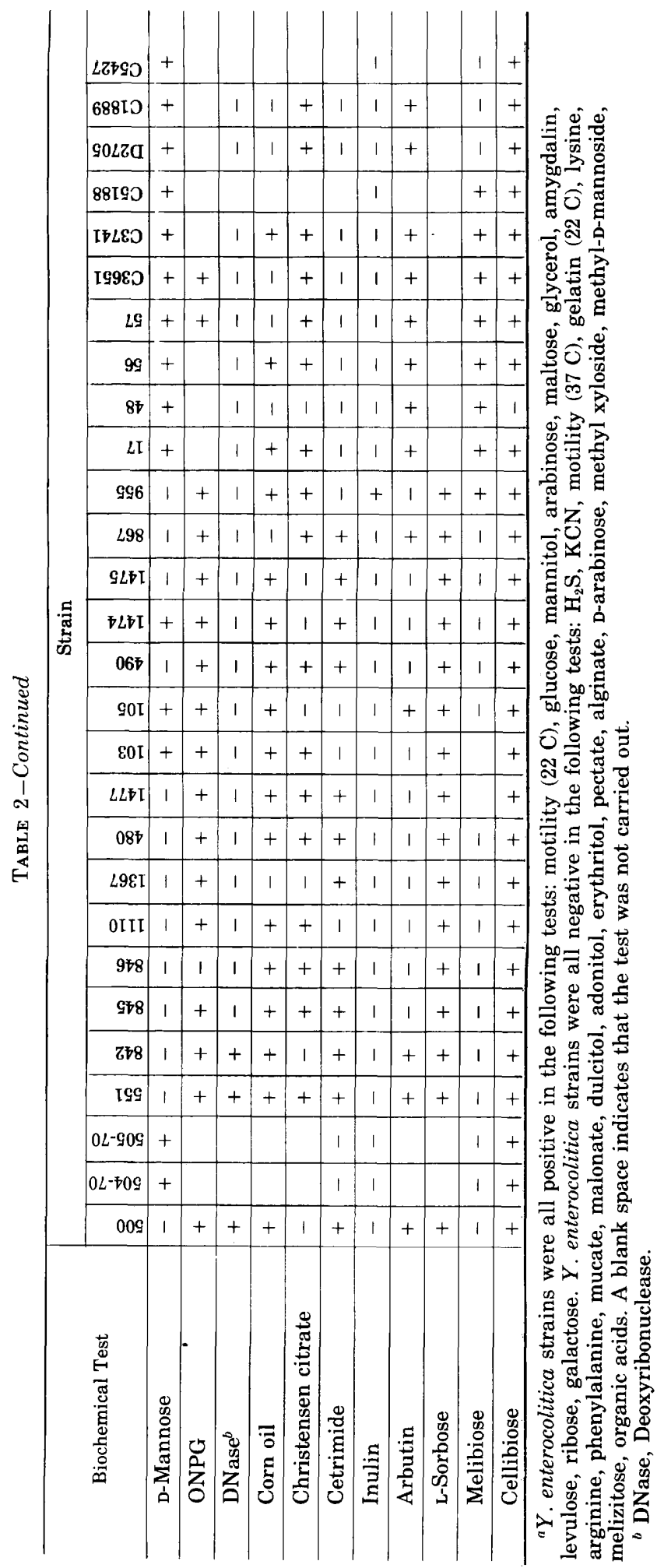


TABLE 3. Biochemical reactions of Yersinia pseudotuberculosis"

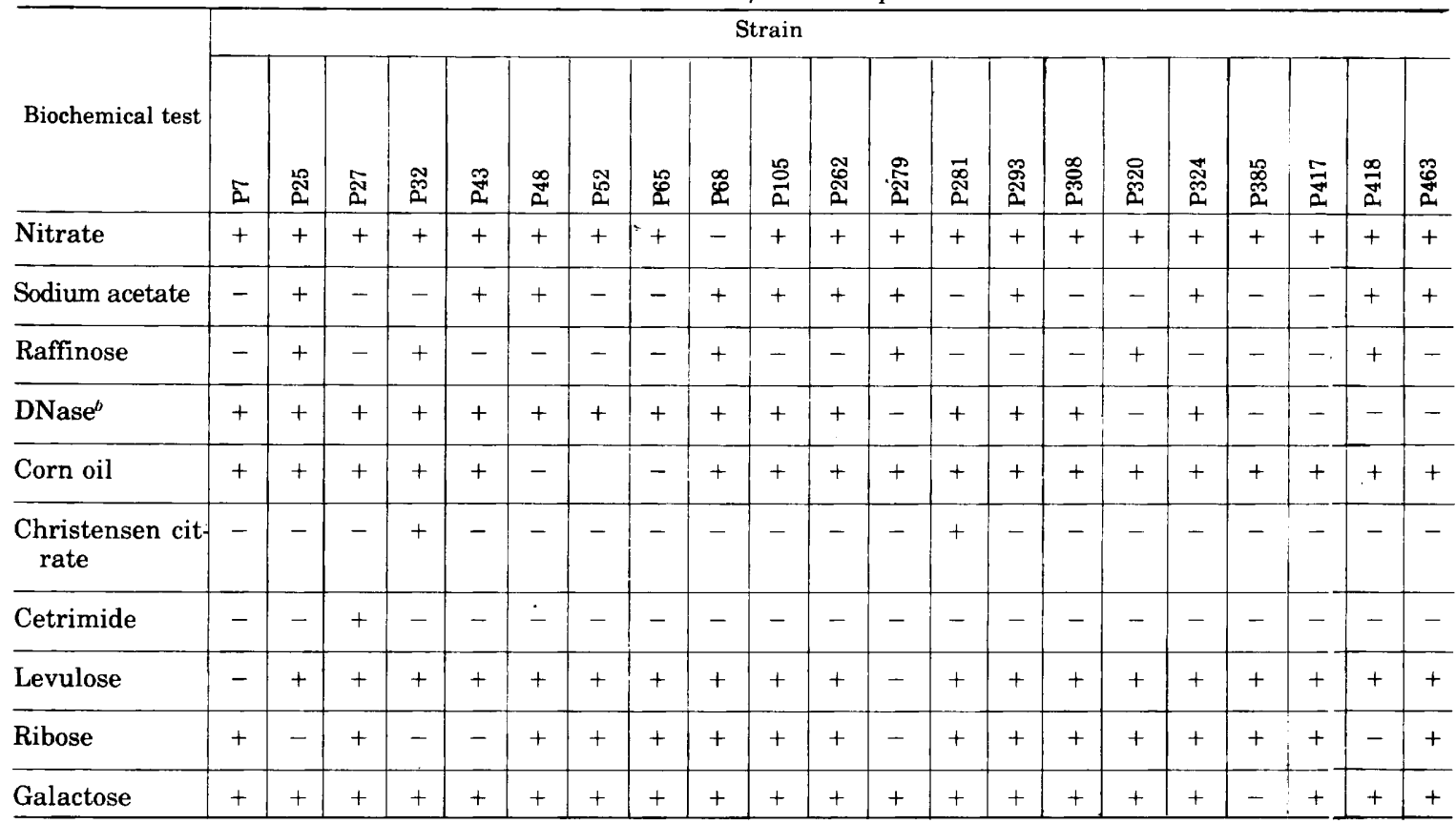

${ }^{a}$ Y.pseudotuberculosis strains were all positive in the following tests: urease, methyl red, motility (22 C), glucose, mannitol, salicin, arabinose, rhamnose, maltose, xylose, trehalose, glycerol, esculin, mannose, o-nitrophenyl- $\beta$-D-galactopyranoside, arbutin. They were all negative in the following tests: $\mathrm{H}_{2} \mathrm{~S}$, indole, Voges-Proskauer, Simmons citrate, KCN, motility (37 C), gelatin (22 C), lysine, arginine, ornithine, phenylalanine, mucate, malonate, lactose, sucrose, dulcitol, adonitol, inositol, sorbitol, cellibiose, $\alpha$-methyl glucoside, erythritol, amygdalin, pectate, alginate, inulin, sorbose, D-arabinose, methyl xyloside, methyl D-mannoside, melizitose, organic acids. Blank space indicates that the test was not carried out.

${ }^{b}$ DNase, Deoxyribonuclease.

between distantly related species of Enterobacteriaceae (4). Results similar to those shown in Table 4 were also obtained when two other typical strains of $Y$. enterocolitica, 497-70 and C5427 (see Table 2), were used as reference strains.

The reactions for indole, D-xylose, esculin, and salicin, respectively, were designated as positive $(+)$ or negative $(-)$ for the $Y$. enterocolitica strains used in Tables 4 through 6 . Many authors $(19,26$; G. Wauters, Ph.D. thesis) placed strains of $Y$. enterocolitica into either four or five biogroups based primarily on these reactions, but other investigators $(10,36$; J. Feeley, personal communication) did not use this biogrouping scheme. Knapp and Thal (19) recognized four biogroups, within which were the following patterns for indole, xylose, esculin, and salicin, respectively:,-----+-- , ,++--++++ , and -++- . We obtained a greater variety in the patterns of indole, xylose, esculin, and salicin reactions, observing ,$-+-- \quad-++-, \quad--++, \quad-+-+,---+$,
,,,-+++++--++-++++- , and ++++ (Tables 2 and 3). Many of the strains employed by Knapp and Thal (19) and those used in the present study were the same, having come from the culture collection at the Pasteur Institute. The reactions we observed in many of these strains were different from those recorded by Knapp and Thal (19). The reasons for these differences will be discussed below.

Because of these biochemical differences, we chose reference strains of $Y$. enterocolitica known to belong to biogroups 1,2 , and 3 of Knapp and Thal (19). The results obtained with labeled DNA from Y. enterocolitica 211 (biogroup 2) are shown in Table 5. All strains of $Y$. enterocolitica are very closely related (RBR, $60 \mathrm{C}=90$ to $100 \%$; percent $\mathrm{D}=0.0$ to 1.2 ; $\mathrm{RBR}$, $75 \mathrm{C}=84$ to $99 \%$ ). Several strains from each of the six serogroups of $Y$. pseudotuberculosis gave RBR values of 45 to $64 \%$ with $Y$. enterocolitica 211. The percent $\mathrm{D}$ in these reactions was usually between 8 and 13 , and RBR values at $75 \mathrm{C}$ decreased significantly. Relatedness be- 
TABLE 4. Relative binding ratios and divergences of DNA from $Y$. enterocolitica 501-70

\begin{tabular}{|c|c|c|c|}
\hline Source of unlabeled DNA ${ }^{a}$ & $\mathrm{RBR}, b 60 \mathrm{C}$ & $\mathrm{D},{ }^{c} 60 \mathrm{C}$ & $\mathrm{RBR}, 75 \mathrm{C}$ \\
\hline Yersinia enterocolitica $501-70-+-+^{d}$ & 100 & 0.0 & 100 \\
\hline Y. enterocolitica $495-70++--$ & 99 & 1.2 & 96 \\
\hline Y. enterocolitica $496-70-+-+$ & 98 & 0.5 & 93 \\
\hline$Y$. enterocolitica $497-70++--$ & 92 & 0.8 & 100 \\
\hline$Y$. enterocolitica $498-70++-+$ & 96 & 0.6 & \\
\hline$Y$. enterocolitica $499-70++-+$ & 99 & 0.8 & 90 \\
\hline$Y$. enterocolitica $500-70-+-+$ & 88 & 2.0 & 83 \\
\hline Y. enterocolitica $502-70---+$ & 85 & 1.0 & 82 \\
\hline$Y$. enterocolitica $503-70+-++$ & 90 & 0.0 & \\
\hline Y. enterocolitica $504-70++++$ & 87 & 2.4 & 83 \\
\hline Y. enterocolitica $505-70++++$ & 87 & 1.6 & \\
\hline Y. enterocolitica $506-70++--$ & 98 & 0.0 & 94 \\
\hline Y. enterocolitica $\mathrm{C} 1889++-+$ & 100 & 0.2 & \\
\hline Y. enterocolitica $\mathrm{C} 5427++-+$ & 94 & 0.0 & 93 \\
\hline$Y$. pestis & 43 & 15.2 & 11 \\
\hline Red Mouth 2396-61 & 31 & 15.5 & \\
\hline Red Mouth 2514-63 & 31 & 15.1 & 8 \\
\hline Red Mouth 4535-69 & 31 & 14.1 & \\
\hline Red Mouth 5972-61 & 29 & 15.5 & 6 \\
\hline Serratia marcescens SM6 red & 25 & & 6 \\
\hline Salmonella typhimurium LT2 & 21 & & 2 \\
\hline Escherichia coli $\mathrm{K}-12$ & 20 & 12.7 & 3 \\
\hline Enterobacter aerogenes $1627-66$ & 20 & & 5 \\
\hline Klebsiella pneumoniae 2 & 19 & & 4 \\
\hline Enterobacter hafniae 932-68 & 19 & & 3 \\
\hline Pectobacterium carotovorum 495 & 19 & & \\
\hline Shigella sonnei virulent & 18 & & \\
\hline Enterobacter cloacae 1347-71 & 18 & & \\
\hline E. cloacae $256-4$ & 18 & & 3 \\
\hline Citrobacter freundii $\mathbf{8 0 - 5 8}$ & 18 & & 4 \\
\hline Enterobacter agglomerans 14589 & 17 & & \\
\hline Proteus morganii & 17 & & 1 \\
\hline Arizona hinshawii 1 & 15 & & \\
\hline Erwinia amylovora EA178 & 15 & & \\
\hline Edwardsiella tarda $1483-59$ & 15 & & \\
\hline E. $\operatorname{tarda} 3979-60$ & 10 & & \\
\hline Proteus mirabilis 1 & 10 & & \\
\hline
\end{tabular}

${ }^{a}$ The + or - symbols indicate positive or negative reactions for indole, xylose, esculin, and salicin, respectively.

${ }^{b} \mathrm{RBR}$, Relative binding ratio $=\%$ (heterologous DNA bound to $\left.\mathrm{HA}\right) /($ homologous DNA bound to HA) $\times$ 100. RBR is a convenient way to express percent relatedness.

c $\mathrm{D}$, Percent divergence. $\mathrm{D}$ is calculated on the assumption that a 1-degree $\mathrm{C}$ decrease in thermal stability of a heterologous DNA duplex compared to that of the homologous DNA duplex is caused by each $1 \%$ of the bases within the duplex that are unpaired (21). For example, consider organisms A and B which are 50\% related $(\mathrm{RBR}=50 \%)$. The thermal stability of an A-A duplex is $92 \mathrm{C}$, and the thermal stability of an A-B duplex is $77 \mathrm{C}$; the percent divergence (D) of the related DNA is 15.0.

${ }^{d}$ Binding of DNA from $Y$. enterocolitica $501-70$ to $\mathrm{HA}$ was $79 \%$ after incubation at $60 \mathrm{C}$ and $78 \%$ after incubation at $75 \mathrm{C}$. The thermal stability of DNA bound to $\mathrm{HA}$ at $60 \mathrm{C}$ was $92.3 \mathrm{C}$.

tween Y. enterocolitica and 15 strains of Pasteurella multocida was between 6 and 13\%. Similar patterns of relatedness between $Y$. enterocolitica, $Y$. pseudotuberculosis, and $P$. multocida were observed when $Y$. enterocolitica strains 614 (biogroup 1) and 106 (biogroup 3) were used as reference strains.

We next employed two grossly atypical strains of $Y$. enterocolitica as the sources of reference DNA. These are strain $178(--++)$, which is nitrate negative, ornithine negative, sorbitol negative, trehalose negative, and sorbose negative, and strain $48(++++$, biogroup 4 , which is methyl red positive, citrate positive, rhamnose positive, cellibiose negative, and melibiose positive. We tested relatedness between these strains and members of all four biogroups (Table 6). Strain 178 was highly related to all typical (by hybridization) $Y$. enterocolitica (Table 6, starting with strain 135), but had RBR values of only 55 to $67 \%$ with all strains from biogroup 4 (strain 48 through 
TABLE 5. Relative binding ratios and divergences of DNA from $Y$. enterocolitica 211

\begin{tabular}{|c|c|c|c|}
\hline Source of unlabeled DNA ${ }^{a}$ & $\mathrm{RBR},{ }^{a} 60 \mathrm{C}$ & $\mathrm{D},{ }^{a} 60 \mathrm{C}$ & RBR, $75 \mathrm{C}$ \\
\hline Yersinia enterocolitica $211-++-^{b}$ & 100 & 0.0 & 100 \\
\hline Y. enterocolitica $373-+--$ & 100 & & \\
\hline Y. enterocolitica $175--++$ & 99 & & 99 \\
\hline$Y$. enterocolitica $135-+-+$ & 97 & & 98 \\
\hline Y. enterocolitica $160-+++$ & 97 & 0.2 & \\
\hline Y. enterocolitica $97+++-$ & 96 & 0.4 & 98 \\
\hline Y. enterocolitica $75--++$ & 95 & 0.2 & \\
\hline Y. enterocolitica $614--++$ & 94 & & 98 \\
\hline Y. enterocolitica $39-+--$ & 94 & 0.0 & \\
\hline Y. enterocolitica $64-++-$ & 94 & & 97 \\
\hline Y. enterocolitica $96-+++$ & 92 & & \\
\hline Y. enterocolitica $161++++$ & 92 & 1.2 & 87 \\
\hline Y. enterocolitica $107+++-$ & 90 & & \\
\hline Y. enterocolitica $501-70-+-+$ & 90 & & 84 \\
\hline Yersinia pseudotuberculosis P25 (5) & 64 & 8.6 & \\
\hline Y. pseudotuberculosis P62 (2) & 62 & 5.4 & 41 \\
\hline Y. pseudotuberculosis P43 (3) & 61 & 10.4 & 33 \\
\hline Y. pseudotuberculosis P417 (5) & 61 & & \\
\hline Y. pseudotuberculosis P262 (1) & 59 & & \\
\hline Y. pseudotuberculosis P324 (4) & 59 & & 26 \\
\hline Y. pseudotuberculosis P65 (2) & 58 & & \\
\hline Y. pseudotuberculosis P279 (4) & 57 & & \\
\hline Y. pseudotuberculosis P418 (5) & 56 & & 16 \\
\hline Y.pseudotuberculosis P320 (1) & 55 & 10.6 & 17 \\
\hline Y. pseudotuberculosis P7 (2) & 55 & & 16 \\
\hline Y. pseudotuberculosis P48 (2) & 55 & 8.6 & \\
\hline Y. pseudotuberculosis P293 (1) & 54 & & \\
\hline Y. pseudotuberculosis P68 (2) & 54 & & 13 \\
\hline Y. pseudotuberculosis P32 (4) & 54 & 12.6 & 28 \\
\hline Y.pseudotuberculosis P463 (6) & 54 & & \\
\hline Y. pseudotuberculosis P105 (3) & 53 & & \\
\hline Y. pseudotuberculosis P27 (3) & 52 & 12.9 & \\
\hline Y. pseudotuberculosis 23207 (?) & 52 & & \\
\hline Y.pseudotuberculosis P281 (1) & 49 & 11.1 & 11 \\
\hline Y. pseudotuberculosis 4284 (?) & 47 & 13.7 & 11 \\
\hline Y. pseudotuberculosis 907 (?) & 47 & 13.6 & 10 \\
\hline Y. pseudotuberculosis 913 (?) & 45 & 13.3 & \\
\hline Salmonella typhimurium LT2 & 26 & 16.8 & 2 \\
\hline Escherichia coli K-12 & 24 & 17.0 & 2 \\
\hline Proteus mirabilis 1 & 19 & & 3 \\
\hline Proteus vulgaris $\mathrm{PRI}$ & 13 & & 1 \\
\hline Pasteurella multocida 6530 & 13 & & 4 \\
\hline P. multocida 10544 & 12 & & \\
\hline P. multocida 12948 & 12 & & 2 \\
\hline P. multocida 6587 & 11 & & \\
\hline P. multocida 15742 & 11 & & \\
\hline P. multocida 15743 & 10 & & \\
\hline P. multocida 7987 & 10 & & 1 \\
\hline P. multocida 12947 & 9 & & \\
\hline P. multocida 9658 & 8 & & 2 \\
\hline P. multocida 8369 & 8 & & \\
\hline P. multocida 6535 & 7 & & \\
\hline P. multocida 7707 & 6 & & 0 \\
\hline P. multocida 7773 & 6 & & \\
\hline P. multocida 7960 & 6 & & \\
\hline P. multocida 9556 & 6 & & \\
\hline
\end{tabular}

\footnotetext{
"See footnotes $a, b, c$ of Table 4 for explanation.

${ }^{b}$ The binding of DNA from Y. enterocolitica 211 to $\mathrm{HA}$ was $79 \%$ in $60 \mathrm{C}$ reactions and $82 \%$ in $75 \mathrm{C}$ reactions. The thermal stability of DNA bound to $\mathrm{HA}$ at $60 \mathrm{C}$ was $90.6 \mathrm{C}$.

c The numbers in parentheses next to the $Y$. pseudotuberculosis strains indicate the serogroups to which the strains belong.
}

strain 105). Strain 48 (biogroup 4) had high RBR values ( 83 to $98 \%$ ) with 6 of the 10 biogroup 4 strains but had RBR values of only 40 to $60 \%$ with the four remaining biogroup 4 strains (biogroup 4 strains are strain 48 through strain 105). Strain 48 was $60 \%$ related to typical strains of $Y$. enterocolitica, 46 to $52 \%$ related to $Y$. pseudotuberculosis, and 7 to $13 \%$ related to $P$. multocida.

The biochemical profiles of biogroup 4 strains fell into three groups (Table 2). One of these groups was sucrose negative (strains 103, 105, 
TABLE 6. Relative binding ratios and divergences of DNA from Y. enterocolitica 178 and $Y$. enterocolitica 48

\begin{tabular}{|c|c|c|c|c|c|}
\hline \multirow{2}{*}{ Source of unlabeled DNA ${ }^{a}$} & \multicolumn{2}{|c|}{ Y. enterocolitica 178} & \multicolumn{3}{|c|}{$\bar{Y}$. enterocolitica 48} \\
\hline & $\mathrm{RBR},{ }^{a} 60 \mathrm{C}$ & $\mathrm{D},{ }^{a} 60 \mathrm{C}$ & $\mathrm{RBR}, 60 \mathrm{C}$ & $\mathrm{D}, 60 \mathrm{C}$ & RBR, $75 \mathrm{C}$ \\
\hline Yersinia enterocolitica $\overline{178--++^{b}}$ & 100 & 0.0 & & & \\
\hline$Y$. enterocolitica $48++++^{b}$ & 61 & 12.8 & 100 & 0.0 & 100 \\
\hline Y. enterocolitica $17++++$ & 58 & 11.9 & 95 & 0.5 & 97 \\
\hline Y. enterocolitica $57++++$ & 56 & & 98 & 0.0 & 100 \\
\hline Y. enterocolitica $56++++$ & 55 & 12.5 & 91 & 1.1 & 87 \\
\hline$Y$. enterocolitica $955++++$ & 63 & 10.5 & 92 & 1.7 & \\
\hline Y. enterocolitica $867++++$ & 62 & 10.1 & 60 & 11.4 & 19 \\
\hline Y. enterocolitica $\mathrm{C} 3741++++$ & & & 93 & & 94 \\
\hline Y. enterocolitica $\mathrm{C} 3651++++$ & & & 85 & & 90 \\
\hline Y. enterocolitica $\mathrm{C} 5188++++$ & & & 83 & & 75 \\
\hline Y. enterocolitica D2705 ++++ & & & 60 & & 31 \\
\hline$Y$. enterocolitica $103-+++$ & 67 & & 48 & 12.6 & 17 \\
\hline Y. enterocolitica $490++++$ & 59 & 8.9 & & & \\
\hline$Y$. enterocolitica $105++++$ & 55 & 9.4 & 42 & 15.0 & 17 \\
\hline Y. enterocolitica $135-+-+$ & 97 & & & & \\
\hline Y. enterocolitica $175--++$ & 96 & 0.0 & & & \\
\hline Y. enterocolitica $373-+--$ & 94 & 0.4 & & & \\
\hline Y. enterocolitica $75--++$ & 93 & 0.0 & & & \\
\hline Y. enterocolitica $106++++$ & 88 & 2.3 & & & \\
\hline Y. enterocolitica $107+++-$ & 88 & 1.7 & & & \\
\hline Y. enterocolitica $1477++++$ & 87 & & & & \\
\hline$Y$. enterocolitica $842++++$ & 86 & & & & \\
\hline Y. enterocolitica $123++++$ & 85 & 0.5 & & & \\
\hline Y. enterocolitica $500-+-+$ & 85 & 1.0 & & & \\
\hline$Y$. enterocolitica $845++++$ & 81 & 3.9 & & & \\
\hline$Y$. enterocolitica $846++++$ & & & 56 & & \\
\hline$Y$. enterocolitica $495-70++--$ & & & $58^{1}$ & & \\
\hline Y. enterocolitica $496-70-+-+$ & & & 61 & 10.9 & \\
\hline Y. pseudotuberculosis P262 & & & 52 & & \\
\hline Y. pseudotuberculosis P320 & & & 50 & & \\
\hline Y. pseudotuberculosis P25 & & & 49 & & \\
\hline Y. pseudotuberculosis P62 & & & 48 & & \\
\hline Y. pseudotuberculosis $\mathrm{P} 105$ & & & 46 & & \\
\hline Pasteurella multocida 10544 & & & 13 & & \\
\hline P. multocida 6530 & & & 11 & & \\
\hline P. multocida 7960 & & & 8 & & \\
\hline P. multocida 7773 & & & 7 & & \\
\hline
\end{tabular}

${ }^{a}$ See footnotes $a, b, c$ of Table 4 for explanation.

${ }^{b}$ The binding of DNA from $Y$. enterocolitica 178 to $\mathrm{HA}$ in $60 \mathrm{C}$ reactions was $77 \%$, and the thermal stability of DNA bound to HA was $91.3 \mathrm{C}$. The binding of $Y$. enterocolitica 48 to HA averaged $65 \%$ in 60 and $75 \mathrm{C}$ reactions. The thermal stability of this bound DNA was $91.6 \mathrm{C}$.

$490,1474,1475)$. The other two groups were rhamnose positive. One of the rhamnose-positive groups (strains 867, D2705) was raffinose negative, melibiose negative, and $\alpha$-methyl glucoside negative; the other was melibiose positive, $\alpha$-methyl glucoside positive, and usually delayed positive for raffinose (strains 17 , 48, 56, 57, C1889, C3651, C3741). DNA relatedness data indicated that the two groups of rhamnose-positive strains belong to different hybridization groups, each of which was distinct from typical $Y$. enterocolitica strains. The sucrose-negative strains are in a hybridization group separate from both typical $Y$. enterocolitica and from strains positive for rhamnose, $\alpha$-methyl glucoside, and melibiose. Since neither a sucrose-negative strain nor a rham- nose-positive, melibiose-negative, $\alpha$-methyl glucoside strain has been used as a reference strain, we cannot yet assign them to different hybridization groups.

$Y$. pseudotuberculosis strains formed the only highly related hybridization group (Table 7). This was not surprising in view of the extremely similar biochemical profiles of these strains (Table 3). RBR values between $Y$. pseudotuberculosis strain P105 and typical strains of $Y$. enterocolitica were about 40 to $50 \%$.

Two ranges of $\mathrm{RBR}$ and $\mathrm{D}$ values occurred between strain P105 and strains of $P$. multocida (Table 7). One group of strains exhibited RBR values of 7 to $13 \%$ with P105. RBR values fell to $3 \%$ or less in $75 \mathrm{C}$ reactions. The $\mathrm{D}$ in these reactions was greater than $10 \%$. The sec- 
TABLE 7. Relative binding ratios and divergences of DNA from $Y$. pseudotuberculosis P105

\begin{tabular}{|c|c|c|c|}
\hline Source of unlabeled DNA & $\mathrm{RBR}, a 60 \mathrm{C}$ & $\mathrm{D},{ }^{a} 60 \mathrm{C}$ & $\mathrm{RBR}, 75 \mathrm{C}$ \\
\hline Yersinia pseudotuberculosis $\mathrm{P} 105^{b}$ & 100 & 0.0 & 100 \\
\hline Y. pseudotuberculosis P281 & 96 & 1.1 & 85 \\
\hline Y. pseudotuberculosis P7 & 95 & 0.0 & 97 \\
\hline Y. pseudotuberculosis P262 & 94 & 0.0 & 88 \\
\hline Y. pseudotuberculosis $\mathrm{P} 62$ & 93 & 0.3 & 92 \\
\hline Y. pseudotuberculosis $\mathrm{P} 65$ & 93 & 0.2 & 93 \\
\hline Y. pseudotuberculosis 23207 & 93 & & \\
\hline Y. pseudotuberculosis $\mathrm{P} 43$ & 92 & 0.0 & 90 \\
\hline Y. pseudotuberculosis $\mathrm{P} 293$ & 92 & 0.3 & 96 \\
\hline Y. pseudotuberculosis $\mathrm{P} 320$ & 92 & 0.0 & 90 \\
\hline Y. pseudotuberculosis $\mathrm{P} 48$ & 91 & 0.6 & 89 \\
\hline Y. pseudotuberculosis $\mathrm{P} 68$ & 91 & 0.3 & 90 \\
\hline Y. pseudotuberculosis $\mathrm{P} 385$ & 89 & & \\
\hline Y. pseudotuberculosis $\mathbf{P 4 6 3}$ & 89 & & 99 \\
\hline Y. pseudotuberculosis $\mathrm{P} 27$ & 88 & & 97 \\
\hline Y. pseudotuberculosis $\mathbf{P 4 1 8}$ & 88 & & 88 \\
\hline Y. pseudotuberculosis P407 & 87 & & \\
\hline Y. pseudotuberculosis P417 & 87 & 0.0 & 92 \\
\hline Y.pseudotuberculosis P32 & 85 & 0.5 & 84 \\
\hline Y. pseudotuberculosis P324 & 85 & 0.0 & 80 \\
\hline Y. pseudotuberculosis 4284 & 83 & 0.7 & 96 \\
\hline Y. pseudotuberculosis 913 & 83 & & \\
\hline Y. pseudotuberculosis P25 & 81 & 1.3 & 71 \\
\hline Y. pseudotuberculosis P279 & 81 & 1.0 & 85 \\
\hline Y. enterocolitica 175 & 53 & 9.7 & 19 \\
\hline$Y$. enterocolitica 64 & 48 & 10.8 & 13 \\
\hline Y. enterocolitica 373 & 46 & 12.1 & 13 \\
\hline Y. enterocolitica 75 & 45 & 13.0 & 18 \\
\hline Y. enterocolitica 135 & 43 & 9.9 & 14 \\
\hline Y. enterocolitica 107 & 42 & 10.5 & 15 \\
\hline Y. enterocolitica 37 & 42 & 11.7 & 14 \\
\hline Pasteurella multocida 12947 & 32 & & \\
\hline P. multocida 6587 & 27 & 2.9 & 18 \\
\hline P. multocida 6530 & 26 & 1.7 & 17 \\
\hline P. multocida 12948 & 26 & 2.7 & 19 \\
\hline P. multocida 10544 & 24 & 4.5 & 13 \\
\hline$P$, multocida 15742 & 17 & 2.7 & 13 \\
\hline P. multocida 7987 & 17 & & \\
\hline P. multocida 8369 & 13 & & \\
\hline P. multocida 6535 & 11 & & 3 \\
\hline P. multocida 7773 & 10 & & \\
\hline P. multocida 7987 & 9 & & 1 \\
\hline P. multocida 7707 & 7 & 13.2 & \\
\hline P. multocida 9658 & 7 & & \\
\hline Red Mouth 2514-63 & 30 & & 7 \\
\hline Red Mouth 2396-61 & 29 & 15.1 & 8 \\
\hline Red Mouth 5972-61 & 29 & 15.0 & 7 \\
\hline Enterobacter cloacae 121-65 & 22 & & 4 \\
\hline Citrobacter freundii $\mathbf{8 0 - 5 8}$ & 22 & & 3 \\
\hline Escherichia coli $\mathrm{K}-12$ & 21 & 14.0 & 3 \\
\hline Serratia marcescens SM6, red & 19 & & 1 \\
\hline Shigella flexneri 2a 24570 & 18 & & 5 \\
\hline Klebsiella pneumoniae 2 & 17 & & 4 \\
\hline Enterobacter agglomerans 1599-71 & 17 & & 3 \\
\hline Salmonella typhimurium LT2 & 15 & & 2 \\
\hline Edwardsiella tarda $3979-60$ & 15 & & 3 \\
\hline Enterobacter aerogenes 1627-66 & 14 & & 4 \\
\hline Arizona hinshawii 1 & 14 & & 1 \\
\hline Erwinia amylovora EA 178 & 11 & & 4 \\
\hline Proteus mirabilis 1 & 11 & & \\
\hline
\end{tabular}

\footnotetext{
${ }^{a}$ See footnotes $b$ and $c$ of Table 4 for explanation.

- The binding of DNA from $Y$. pseudotuberculosis $\mathrm{P} 105$ to $\mathrm{HA}$ in $60 \mathrm{C}$ and $75 \mathrm{C}$ reactions was $71 \%$. The thermal stability of bound DNA was $89.7 \mathrm{C}$.
}

ond group of $P$. multocida strains had RBR values of 17 to $32 \%$ with P105. What is particularly noteworthy about these strains is that RBR values remained relatively high in $75 \mathrm{C}$ reactions (13 to $18 \%$ ) and that $\mathrm{D}$ values were only 1.7 to $4.5 \%$. The indication is that the related sequences between $Y$. pseudotuberculosis and this second group of $P$. multocida 
strains contain only a small percentage of unpaired bases.

Other enteric bacteria exhibit the same level of relatedness to $Y$. pseudotuberculosis (mainly 15 to $20 \%$ ) as seen previously to $Y$. enterocolitica (Table 4). RBR values between Red Mouth bacteria and $Y$. pseudotuberculosis $(30 \%)$ are also similar to those observed between Red Mouth bacteria and Y. enterocolitica (Table 4).

To assess the potential application of DNA hybridization in a diagnostic laboratory, we tested three suspected $Y$. enterocolitica cultures sent to the Center for Disease Control, Atlanta, Ga., for identification. The cultures were grown and DNA was isolated by a rapid modification of our standard techniques (3). All three were confirmed as $Y$. enterocolitica in reactions with a typical strain of $Y$. enterocolitica ( $\mathrm{RBR}=70 \%$ or higher in $75 \mathrm{C}$ reactions). Control reactions with strains of Escherichia coli, Salmonella, Klebsiella, and Citrobacter were negative (RBR $=10 \%$ or lower).

\section{DISCUSSION}

In 1972 the Subcommittee on the Taxonomy of Pasteurella, Yersinia, and Francisella (25) designated $Y$. enterocolitica strains $39,62,160$, 161 , and 373 as reference strains. Their report stated that "strains whose biochemical characteristics are in good agreement with those reference strains are to be considered as belonging to Yersinia enterocolitica." These strains (62 was not tested) were all highly related and were in the hybridization group composed of typical $Y$. enterocolitica strains (Table 5).

Knapp and Thal (19) placed Y. enterocolitica and $Y$ enterocolitica-like organisms into four groups according to reactions for indole, xylose, esculin, and salicin. Group 1 is ---- or -+-- for these reactions. Group 2 is ++-- , and group 3 is ++++ . Group 4 is composed of atypical strains. The patterns given are ++++ , lactose positive and rhamnose positive; - - - , ornithine decarboxylase negative; and -++- , lactose positive, sucrose negative. They stated that groups 3 and 4 should not be included in $Y$. enterocolitica. They also stated that, if indole-positive variants belong to a separate species, $Y$. enteritidis may be used for indole-negative strains and $Y$. enterocolitica for indole-positive strains.

Groups 1, 2, and 3 (Tables 4-6) all belonged to the same DNA relatedness group. Therefore, we saw no reason to separate indole-positive and indole-negative strains and no basis for excluding group 3 strains from $Y$. enterocolit$i c a$. The group 4 strains tested were less closely related to typical $Y$. enterocolitica (Table 6). In fact, they definitely formed two (and probably a third) relatedness groups separate from that of typical $Y$. enterocolitica. Sucrose-negative strains varied in the ability to ferment lactose (Tables 2 and 3 ). These strains are acetoin negative (35). Only one of the three sucrose-negative strains used in hybridization experiments was lactose positive. The same held true for rhamnose-positive strains where both positive and negative lactose reactions were observed (Tables 2 and 3). The rhamnose-positive strains formed two relatedness groups (Table 6), one of which could be differentiated by $\alpha$-methyl glucoside, melibiose, and usually raffinose tests.

All strains of $Y$. pseudotuberculosis belonged to one DNA relatedness group. This finding correlates well with the rather homogeneous biochemical profiles of these strains (Tables 2 and 3 ; references $24,37,38$ ). $Y$. pseudotuberculosis (Table 7) was 40 to $60 \%$ related to $Y$. enterocolitica. Both Yersinia species showed low but significant relatedness to other members of Enterobacteriaceae. It is difficult to use hybridization data for taxonomic purposes at the family level; suffice it to say that this level of relatedness seems to be present as a "core" within distantly related members of Enterobacteriaceae (4).

$Y$. enterocolitica strains had RBR values of 6 to $13 \%$ to $P$. multocida (Tables 5 and 6 ). $Y$. pseudotuberculosis exhibited RBR values of 7 to $32 \%$ to strains of $P$. multocida (Table 7). These reactions appeared to fall into two ranges of relatedness ( 7 to $13 \%$ and 17 to $32 \%$ ), or even three ranges of relatedness ( 7 to $11 \%, 13$ to $17 \%$ 24 to $32 \%$ ). Of particular interest was that, in reactions where the RBR was 17 to $32 \%$, percent $\mathrm{D}$ was only 1.7 to 4.5 . The low amount of unpaired bases in related sequences was confirmed by the RBR values from $75 \mathrm{C}$ reactions. These values showed that at least two-thirds of the sequences related at $60 \mathrm{C}$ remained stable at the stringent $75 \mathrm{C}$ reassociation criterion. We have not previously seen evidence of closely related sequences in species where overall relatedness was $30 \%$ or less.

Possible explanations for these results are as follows. (i) DNA from the P. multocida strains exhibiting 17 to $32 \%$ relatedness to $Y$. pseudotuberculosis was contaminated with $Y$. pseudotuberculosis DNA. (ii) The labeled Y. pseudotuberculosis DNA was contaminated with DNA from $P$. multocida. (iii) The results are valid and indicate that $P$. multocida strains belong to more than one DNA relatedness group, one of which contains about $15 \%$ of DNA which is conserved with respect to $Y$. pseudotuberculosis DNA. 
The first two possibilities cannot be ruled out, but there is no evidence to support them. If the labeled Y. pseudotuberculosis DNA preparation had been contaminated, we would have expected lower relatedness of unlabeled $Y$. pseudotuberculosis strains to the reference strain. If the $P$. multocida strains that have RBR values of 17 to $32 \%$ to $Y$. pseudotuberculosis are contaminated with $Y$. pseudotuberculosis DNA, we should have also seen somewhat higher relatedness between these strains and $Y$. enterocolitica. This was not the case (Table 5).

One piece of evidence may support the validity of these data. Fredericksen (14) reported that $P$. multocida strains contain different percentages of guanine plus cytosine $(\mathrm{G}+\mathrm{C})$ in their DNA. $G+C$ values of 37,40 , and $43 \mathrm{~mol} \%$ occur. The $\mathrm{G}+\mathrm{C}$ content of $Y$. pseudotuberculosis is 43 to $46 \mathrm{~mol} \%$. It is attractive to theorize that those $P$. multocida strains exhibiting 17 to $32 \%$ relatedness to $Y$. pseudotuberculosis are the $43 \mathrm{~mol} \% \mathrm{G}+\mathrm{C}$ strains.

During this study, biochemical profiles were obtained that were not listed by Knapp and Thal (19). A search of the literature revealed that several groups of investigators have commented upon the difficulty of interpreting certain biochemical reactions given by $Y$. enterocolitica at $37 \mathrm{C}$. This is especially true for indole, xylose, esculin, and salicin for which reactions have been reported as weak, weakly positive, and irregularly positive $(2,10,26)$. A second difficulty is that many positive reactions are not evident at $37 \mathrm{C}$ until 5 to 10 days have elapsed. If tests are discarded after $48 \mathrm{~h}$, the number of negatives will be falsely high (or, if one prefers, cultures held for 1 to 2 weeks will yield a disproportionately high number of positive reactions). It is known that $Y$. enterocolitica is motile at $22 \mathrm{C}$ but not at $37 \mathrm{C}$. The same is true for many biochemical reactions. Therefore, the exact temperature of incubation may well be critical to obtaining a positive or negative reaction. One would expect more positive reactions in a laboratory where the incubator was 33 to $35 \mathrm{C}$ than in a laboratory where the incubator fluctuated between 36 and $38 \mathrm{C}$. In addition, some cultures left at room temperature for an hour or longer might be expected to yield spurious " $37 \mathrm{C}$ " positive reactions. Finally, the method used to carry out a given test may affect the results obtained $(2,37)$.

In 1966 Ritter and Gerloff (28) did hybridizations on single strains of Pasteurella novicida, P. multocida, Francisella tularensis, $Y$. pestis, Y. pseudotuberculosis, Y. philomiragia, and $E$. coli. The Yersinia strains were 6 to $14 \%$ related to the strains of $P$. multocida. Y. pseu- dotuberculosis and $Y$. pestis strains were $87 \%$ related. The $Y$. philomiragia strain was $24 \%$ related to the $Y$. pestis strain, and the $E$. coli strain was $22 \%$ related to the $Y$. pestis strain. We tested a single strain of $Y$. pestis, which was $43 \%$ related to $Y$. enterocolitica 501-70 (Table 4). Further work must be done on $Y$. philomiragia, Pasteurella, and Francisella.

\section{ACKNOWLEDGMENTS}

We are grateful to W. H. Ewing for fruitful discussions and encouragement during this study. We thank J. Feeley for his help and encouragement.

We thank E. Cadet for competent technical assistance.

D. P. Falcão was supported by the Fundação de Ampara a Pesquisa do Estado de São Paulo.

\section{ADDENDUM}

After the submission of this manuscript, a study on DNA hybridization in Yersinia enterocolitica was published (R. L. Moore and R. R. Brubaker, Int. J. Syst. Bacteriol. 25:336-339, 1975). These authors found 18 to $24 \%$ relatedness between $Y$. enterocolitica and $Y$. pseudotuberculosis, and 2 to $16 \%$ relatedness between $Y$. enterocolitica and seven genera other than Yersinia within Enterobacteriaceae. These experiments were carried out in 0.17 $\mathrm{M} \mathrm{NaCl}$ at $64 \mathrm{C}$. Their results are in good agreement with our $75 \mathrm{C}$ data obtained in $0.14 \mathrm{M}$ PB (0.21 M sodium concentration).

\section{REPRINT REQUESTS}

Address reprint requests to: Don J. Brenner, Bldg. 1, Room B311, Center for Disease Control, Atlanta, Ga. 30333.

\section{LITERATURE CITED}

1. Ahvonen, P. 1972. Human yersiniasis in Finland. I. Bacteriology and serology. Ann. Clin. Res. 4:30-38.

2. Bottone, E. J., B. Chester, M. S. Malowany, and J. Allerhand. 1974. Unusual Yersinia enterocolitica isolates not associated with mesenteric lymphadenitis. Appl. Microbiol. 27:858-861.

3. Brenner, D. J. 1974. DNA reassociation for the clinical differentiation of enteric bacteria. Publ. Health Lab. 32:118-130.

4. Brenner, D. J., and S. Falkow, 1971. Molecular relationships among members of the Enterobacteriaceae. Adv. Genet. 16:81-118.

5. Brenner, D. J., G. R. Fanning, K. E. Johnson, R. V. Citarella, and S. Falkow. 1969. Polynucleotide sequence relationships among members of the Enterobacteriaceae. J. Bacteriol. 98:637-650.

6. Brenner, D. J., G. R. Fanning, A. Rake, and K. E. Johnson. 1969. A batch procedure for thermal elution of DNA from hydroxyapatite. Anal. Biochem. 28:447459.

7. Brenner, D. J., A. G. Steigerwalt, G. V. Miklos, and G. R. Fanning. 1973. Deoxyribonucleic acid relatedness among erwiniae and other Enterobacteriaceae: the soft-rot organisms (genus Pectobacterium Waldee). Int. J. Syst. Bacteriol. 23:205-216.

8. Britten, R. J., and D. E. Kohne. 1966. Nucleotide sequence repetition in DNA. Carnegie Inst. Wash. Yearb. 65:78-106.

9. Buchanan, R. E., and N. E. Gibbons (ed.). 1974. Bergey's manual of determinative bacteriology, 8 th ed. The Williams and Wilkins Co., Baltimore.

10. Darland, G., W. H. Ewing, and B. R. Davis. 1975. The 
biochemical characteristics of Yersinia enterocolitica and Yersinia pseudotuberculosis. CDC Publication, Center for Disease Control, Atlanta, Ga.

11. Edwards, P. R., and W. H. Ewing. 1972. Identification of Enterobacteriaceae, 3rd ed. Burgess Publishing Co., Minneapolis, Minn.

12. Ewing, W. H., and B. R. Davis. 1970. Media and tests for differentiation of Enterobacteriaceae. CDC Publication, Center for Disease Control, Atlanta, Ga.

13. Frederiksen, W. 1964. A study of some Yersinia pseudotuberculosis-like bacteria ("Bacterium enterocoliticum" and "Pasteurella X"). Scand. Congr. Pathol. Microbiol. Proc. XIV:103-104.

14. Fredericksen, W. 1973. Pasteurella taxonomy and nomenclature. Contrib. Microbiol. Immunol. 2:170-176.

15. Gurry, J. F. 1974. Acute terminal ileitis and Yersinia infection. Br. Med. J. 2:264-266.

16. Hassig, A., J. Karrer, and F. Pusterla. 1949. Uber Pseudotuberkulose beim Menschen. Schweiz. Med. Wochenschr. 79:971-973.

17. Jansson, E., G. R. Wallgren, and P. Ahvonen. 1968. Yersinia enterocolitica as a cause of acute mesenteric lymphadenitis. Acta Paediatr. Stockholm 57:448-450.

18. Jensen, W. I., C. R. Owen, and W. J. Jellison. 1969. Yersinia philomiragia sp. n., a new member of the Pasteurella group of bacteria, naturally pathogenic for the muskrat (Ondatra zibethica). J. Bacteriol. 100:1237-1241.

19. Knapp, W., and E. Thal. 1973. Differentiation of Yersinia enterocolitica by biochemical reactions. Contrib. Microbiol. Immunol. 2:10-16.

20. McClure, H. M., R. E. Weaver, and A. F. Kaufmann. 1971. Pseudotuberculosis in nonhuman primates: infection with organisms of the Yersinia enterocolitica group. Lab. Anim. Sci. 21:376-382.

21. McConoughy, B. L., and B. J. McCarthy. 1970. Related base sequences in the DNA of simple and complex organisms. VI. The extent of base sequence divergence among the DNAs of various rodents. Biochem. Genet. 4:425-446.

22. McMahon, P. C. 1973. Mapping the chromosome of Yersinia pseudotuberculosis by interrupted mating. J. Gen. Microbiol. 77:61-69.

23. Mair, N. S., H. J. Mair, E. M. Stirk, and J. G. Corson. 1960. Three cases of acute mesenteric lymphadenitis due to Pasteurella pseudotuberculosis. J. Clin. Pathol. 13:432-439.

24. Mollaret, H. H. 1961. Contribution a l'etude des caracteres biochemiques de Pasteurella pseudotuberculosis (Bacille de Malassez et Vignal). Ann. Inst. Pasteur (Paris) 100:685-690.

25. Mollaret, H. H., and W. Knapp. 1972. International Committee on Systematic Bacteriology Subcommit- tee on the Taxonomy of Pasteurella, Yersinia, and Francisella. Int. J. Syst. Bacteriol. 22:401.

26. Nilehn, B. 1969. Studies on Yersinia enterocolitica with special reference to bacterial diagnosis and occurrence in human acute enteric disease. Acta. Pathol. Microbiol. Scand. Suppl. 206:1-48.

27. Niléhn, B., and B. Sjostrom. 1967. Studies on Yersinia enterocolitica: occurrence in various groups of acute abdominal disease. Acta Pathol. Microbiol. Scand. 71:612-628.

28. Ritter, D. B., and R. K. Gerloff. 1966. Deoxyribonucleic acid hybridization among some species of the genus Pasteurella. J. Bacteriol. 92:1838-1839.

29. Ross, A. J., R. R. Rucker, and B. H. Ewing. 1966 Description of a bacterium associated with redmouth disease of rainbow trout (Salmo gairdneri). Can. J. Microbiol. 12:763-770.

30. Schleifstein, J., and M. B. Coleman. 1939. An unidentified microorganism resembling $B$. lignieresi and $P a s-$ teurella pseudotuberculosis, pathogenic for man. N. Y. State J. Med. 39:1749-1753.

31. Smith, J. E., and E. Thal. 1965. A taxonomic study of the genus Pasteurella using a numerical technique. Acta. Pathol. Microbiol. Scand. 64:213-223.

32. Sonnenwirth, A. C. 1970 . Bacteremia with and without meningitis due to Yersinia enterocolitica, Edwardsiella tarda, Comamonas terrigena and Pseudomonas maltophilia. Ann. N. Y. Acad. Sci. 174:488-502.

33. Stevens, M., and N. S. Mair. 1973. A numerical taxonomic study of Yersinia enterocolitica strains. Contrib. Microbiol. Immunol. 2:17-22.

34. Toma, S., and L. Lafleur. 1974. Survey on the incidence of Yersinia enterocolitica infection in Canada. Appl. Microbiol. 28:469-473.

35. Wauters, G. 1973. Correlation between ecology, biochemical behaviour and antigenic properties of Yersinia enterocolitica. Contrib. Microbiol. Immunol. 2:3841.

36. Weaver, R. E., and J. G. Jordan. 1973. Recent human isolates of Yersinia enterocolitica in the United States. Contrib. Microbiol. Immunol. 2:120-125.

37. Wetzler, T. F., and W. T. Hubbert. 1968. Yersinia enterocolitica in North America. Int. Symp. Pseudotuberculosis 9:343-356.

38. Wetzler, T. F., M. N. Rosen, and J. D. Marshall, Jr. 1970. The Pasteurella, the Yersinia and the Francisella, p. 67-81. In C. D. Graber (ed.), Rapid diagnostic methods in medical microbiology. The Williams and Wilkins Co., Baltimore.

39. Winblad, S., B. Nilehn, and N. H. Sternby. 1966. Yersinia enterocolitica (Pasteurella $\mathrm{X}$ ) in human enteric infections. Br. Med. J. 2:1363-1366. 